\title{
Optically tuned poly (3-hexylthiophene-2,5-diyl) P3HT/PCBM (modified fullerene) blend for plastic solar cell
}

\author{
Ishwar Naik $^{1 *}$, Rajashekhar Bhajantri ${ }^{2}$, Lohit Naik ${ }^{2}$, B.S. Patil ${ }^{3}$, Ullas shetti Pragasam ${ }^{1}$, \\ Sunil Rathod ${ }^{4}$, Jagadeesh Naik ${ }^{4}$ \\ ${ }^{1}$ Govt. Arts \& Science College, Karwar, India \\ ${ }^{2}$ Dept. of Physics, Karnatak University, Dharwad, India \\ ${ }^{3}$ Govt. First Grade College Poojgeri, Ankola, India \\ ${ }^{4}$ Dept. of Physics, Mangalore University, Mangalore, India \\ *iknaik@rediffmail.com, rfbhajantri@gmail.com
}

PACS 73.61.Ph; 73.50.Pz; 81.01.Fb; 81.05.ub; 82.35.Cd; 83.80.Tc

DOI 10.17586/2220-8054-2016-7-4-691-694

Nanotechnology-assisted organic electronics is a wide-spread and promising research field at present in the search for an efficient solar cell. The strong absorption spectra of the donor polymer poly[3-hexylthiophene-2,5-diyl)(P3HT) in the visible region and the prominent absorption of the acceptor [6,6]-phenyl $\mathrm{C}_{61}$ butyric acid methyl ester(PCBM) in UV region have proved them to be the most popular donor-acceptor pair for preparing photoactive materials. The active blend, having a broad absorption spectrum, is the primary requisite for an efficient solar cell. The present work is focused on optimizing the photoactive blend of (P3HT)) and PCBM for the maximum absorption of the solar energy. P3HT: PCBM blends of weight ratio 3:1, 1:1 \& 1:3 were prepared in xylene as the solvent and glass coated samples are prepared by solution cast method. Samples were characterized by JASCO UV Vis NIR V 670 spectrometer. P3HT has strong absorption in the visible region, while PCBM has an effective absorption in the UV region with broad tail of absorption extending up to $800 \mathrm{~nm}$. The spectrum for the blend is a superposition of the spectra of the component moieties. The 1:3 blend of P3HT with PCBM has broad spectral sensitivity for absorption and can be used as the best photoactive blend for construction of a plastic solar cell. The energy difference (band gap) between Highest Occupied Molecular Orbital (HOMO) and the Lowest Un-occupied Molecular Orbital (LUMO) of the samples were determined through Tauc's plot. Calculations from Tauc's plot indicated that pure P3HT sample has an onset wavelength of $640 \mathrm{~nm}$ with a band gap of $1.93 \mathrm{eV}$. The onset wavelengths for 3:1 \& 1:1 blends are almost same of about $632 \mathrm{~nm}$ with a band gap of $1.96 \mathrm{eV}$. The optimized blend 1:3 sample has the onset wavelength $653 \mathrm{~nm}$ with the least energy gap $1.9 \mathrm{eV}$.The absorption by the blend can be further enhanced by either dye sensitization or plasmon resonance.

Keywords: P3HT, PCBM, HOMO, LUMO, LSPR.

Received: 5 February 2016

\section{Introduction}

First generation silicon-based solar cells are are hindered by material cost, installation cost, and fabrication complications. Even the second generation thin film solar cells are also not economical [1]. Plastic solar cells are promising because of their low cost, simple processing and flexibility. Since efficiency is the main drawback of these solar cells, the search for an efficient plastic solar cell has become a global challenge at present in the field of plastic electronics. The physics involved in the energy conversion efficiency of polymer cells is a multistep process, including photon absorption, exciton formation and migration, exciton dissociation, charge transport and charge collection [2]. The bulk heterojunction concept of the donor-acceptor system has provided remarkable progress in the efficiency enhancement in this regard. The exciton diffusion mechanism and the charge collection rate have been significantly increased due to the formation of number of hetero P-N junctions in the blend. The photoactive blend must be properly tuned with respect to the choice of donor-acceptor pair as well as their weight percentage combination to have a broad solar spectral response. Among the conducting polymers, P3HT has the absorption spectrum matching well with the strongest solar spectrum and also has good transport properties. The present work is focused on the preparation of an optimized active blend of the widely used donor polymer Poly[3-hexylthiophene-2,5-dily) (P3HT) and N-type acceptor [6,6]-Phenyl $\mathrm{C}_{61}$ butyric acid methyl ester (PCBM), a modified fullerene. The strong absorption spectrum of P3HT in the visible region and the prominent absorption of PCBM in the UV region, along with the broad tail of absorption beyond the visible region are the key factors in selecting them as the donor-acceptor pair. The resulting active blend must show broad spectral absorption extending from the UV to the region beyond the visible for a proper composition between them. Blends of different donor-acceptor weight ratios were prepared in the high boiling point solvent xylene and their glass 
coated samples (solution cast method) were characterized by UV-Visible spectra. The blend showing broad spectral sensitivity for absorption is selected as the best photoactive blend.

\section{Experimental}

The P-type donor polymer P3HT (electronic grade, average mol wt 15000 - 45000, HOMO $5 \mathrm{eV}$, LUMO $3 \mathrm{eV}$ ) and N-type acceptor PCBM (functionalized Fullerene, HOMO 6.1 eV,LUMO $3.7 \mathrm{eV}$ ) were purchased from Sigma Aldrich Corporation. The solvent xylene was obtained from Rankem Chemicals. These chemicals are used as received without further purification. The chemical structures of these donor and acceptor molecules are as shown below.

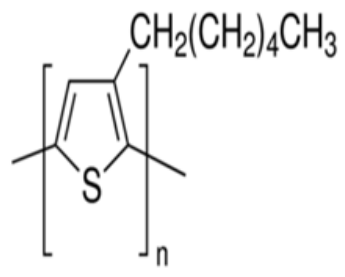

P3HT

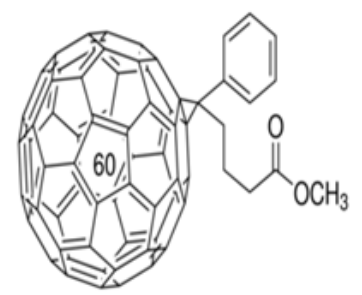

PCBM

$10 \mathrm{mg}$ of P3HT and $10 \mathrm{mg}$ of PCBM were dissolved in $100 \mathrm{ml}$ of xylene in separate beakers and magnetically stirred for $48 \mathrm{hrs}$ at room temperature until clear solutions were formed. The resulting solutions were of concentrations $0.1 \mathrm{mg} / \mathrm{ml}$ each. The solutions were blended with P3HT: PCBM at weight ratios of 3:1, 1:1 \& 1:3 keeping the total weight of the film fixed at $4 \mathrm{mg}$. The mixtures were magnetically stirred for 3 days at room temperature and then transferred to $3 \mathrm{~cm}$ diameter petri-plates, dried at room temperature and then at about $50{ }^{\circ} \mathrm{C}$ in hot air oven. The film composition is as shown in Table 1.

TABLE 1. Weight proportions of blends

\begin{tabular}{|c|c|c|}
\hline P3HT/Xylene (P type) & PCBM/Xylene (N type) & P:N \\
\hline $4 \mathrm{mg}$ & $0 \mathrm{mg}$ & Pure P \\
\hline $3 \mathrm{mg}$ & $1 \mathrm{mg}$ & $3: 1$ \\
\hline $2 \mathrm{mg}$ & $2 \mathrm{mg}$ & $1: 1$ \\
\hline $1 \mathrm{mg}$ & $3 \mathrm{mg}$ & $1: 3$ \\
\hline $0 \mathrm{mg}$ & $4 \mathrm{mg}$ & Pure N \\
\hline
\end{tabular}

\section{Result and discussion}

One of the major problems in polymer solar cells is insufficient absorption in the solar irradiation spectrum. Even the widely studied phenylene-vinylene polymers have band edges at $550 \mathrm{~nm} \mathrm{[2].} \mathrm{However,} \mathrm{the} \mathrm{pure} \mathrm{P3HT}$ film has absorption extending from $300 \mathrm{~nm}$ to $640 \mathrm{~nm}$. The spectrum has two peaks at $520 \mathrm{~nm}$ and $560 \mathrm{~nm}$ with one shoulder around $620 \mathrm{~nm}$, having onset of absorption (the absorption edge) at about $640 \mathrm{~nm}$, matching well with the strongest solar spectrum (Fig. 1(a)). The first two peaks arise from $\pi-\pi^{*}$ transition and the shoulder is due to interchain interactions [2]. Pure PCBM shows the strongest absorption in the UV region with a broad tail of absorption extending up to $800 \mathrm{~nm}$ (Fig. 1(e)). The strong absorption in the UV region arises from HOMO-LUMO (Highest Occupied Molecular Orbital-Lowest Unoccupied Molecular Orbital) transitions [3] or in other words the strongest absorption is attributed to the formation of higher excited singlet states [4]. The spectra are in good agreement with previously reported results. Spectra for all other blends are the superposition of the component spectra. The effect of PCBM in the blend is to reduce the absorption of P3HT in the visible region followed by increase of absorption in UV region and beyond $650 \mathrm{~nm}$. The modification in the spectrum is because of the interaction between PCBM molecules and P3HT polymer chain. Modified fullerene molecules hinder the interaction among the P3HT chains [5]. Although the 3:1 and 1:1 blends have enough absorption in the visible region as indicated Fig. 1(b) and Fig. 1(c) respectively, the absorption coefficient of 1:3 blend is greater in the UV region and beyond $640 \mathrm{~nm}$ as depicted in Fig. 1(d). Relatively, the 1:3 blend has wide spectral sensitivity (Fig. 1(f)) and can be considered as the best photoactive blend among the samples prepared. Pure P3HT sample has onset wavelength of $640 \mathrm{~nm}$ with a band gap of $1.93 \mathrm{eV}$ as calculated from Fig. 2(a). Onset wavelengths for 3:1 \& 1:1 blends are almost same of about $632 \mathrm{~nm}$ with a band gap of $1.96 \mathrm{eV}$ as revealed by Fig. 2(b) and Fig. 2(c). The 
calculations from Tauc's plot shows that the onset wavelength of the optimized blend is 653 nm with band gap $1.9 \mathrm{eV}$ in accordance with Fig. 2(d). The absorption by the blend can be further enhanced by doping with metal nano particles, exploiting Localised Surface Plsmon Resonance (LSPR) or by dye sensitization. Nanodoping/dye sensitization of 1:3 P3HT: PCBM blend and construction of solar cell using the same is our work in progress.

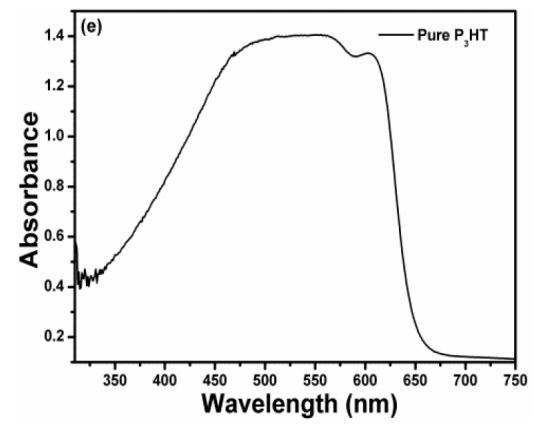

(a)

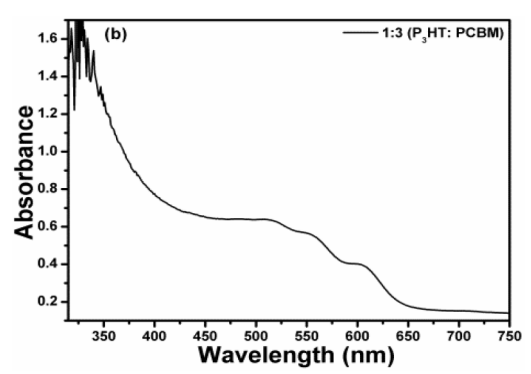

(d)

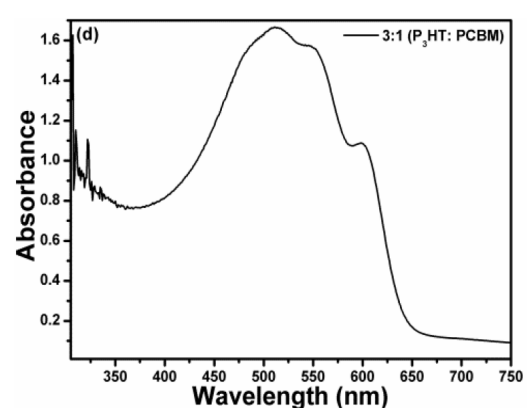

(b)

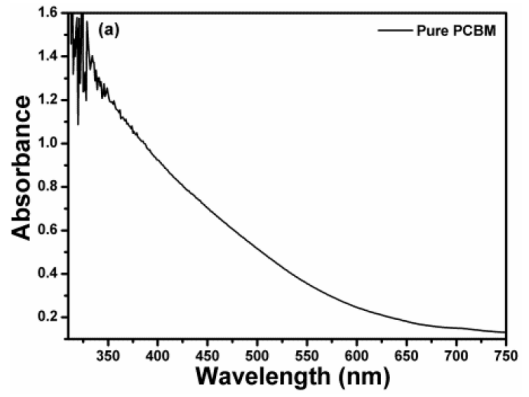

(e)

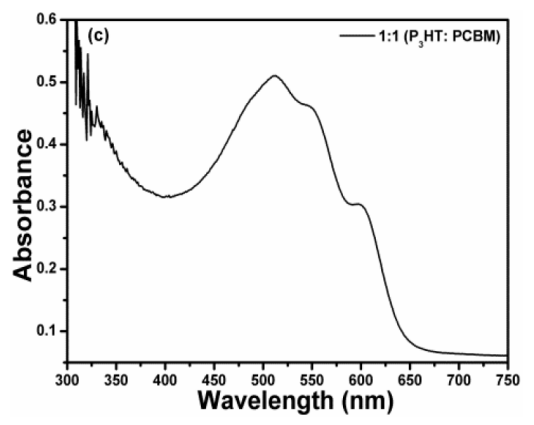

(c)

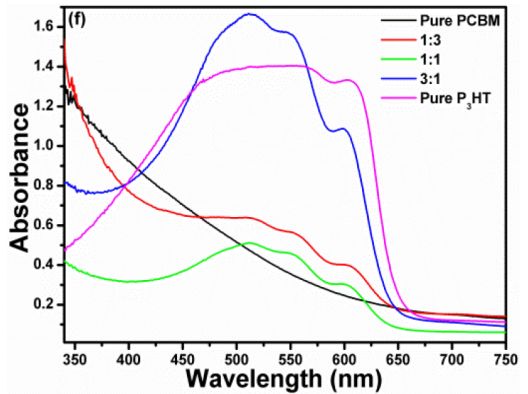

(f)

FIG. 1. Spectrum of pure P3HT film (a); 3:1 (b); 1:1 (c); 1:3 (d) P3HT:PCBM; pure PCBM (e) and overlay spectra of all films (f)

\section{Conclusion}

We have investigated the UV-VISIBLE absorption spectra for 3:1, 1:1, 1:3 blends of P3HT:PCBM mixed p-n junction photoactive material along with their pristine glass coated films. Spectral analysis indicated that increased weight percentage of PCBM in the blend broadened the spectral region of absorption. The 1:3 blend of P3HT:PCBM showed broad spectral absorption and was selected as the best photoactive blend. The optimized blend had the smallest band gap $(1.9 \mathrm{eV})$. By doping the blend with metal oxide nanoparticles or by dye sensitization, the absorption can be further enhanced. Exploiting Plasmon Resonance through nanodoping of the selected blend is an area for future investigation. Additionally, there are plans for dye sensitization of the active blend which are being explored. Finally, we conclude that the 1:3 blend of P3HT:PCBM can be used as a photoactive material for constructing a plastic solar cell and the construction of this solar cell is progressing.

\section{Acknowledgements}

Thanks to UGC for sanctioning the Minor research project entitled 'construction and characterization of an organic solar cell (OPV) devised from a self-made low cost spin coating machine'. Order No. 1419-MRP/1415/KAKA088/UGC-SWRO, dated 04-02-2015. 


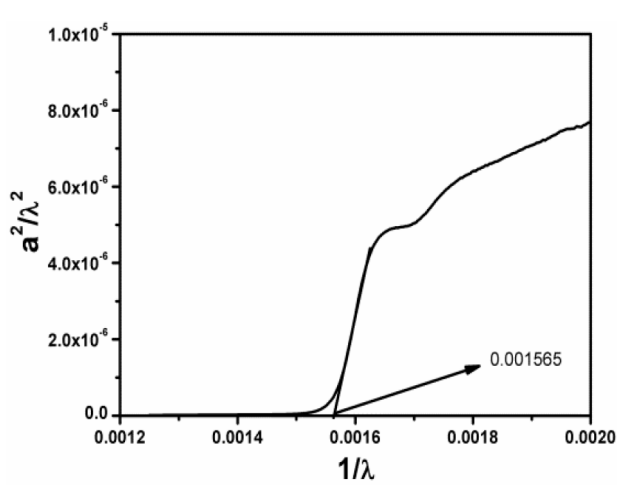

(a)

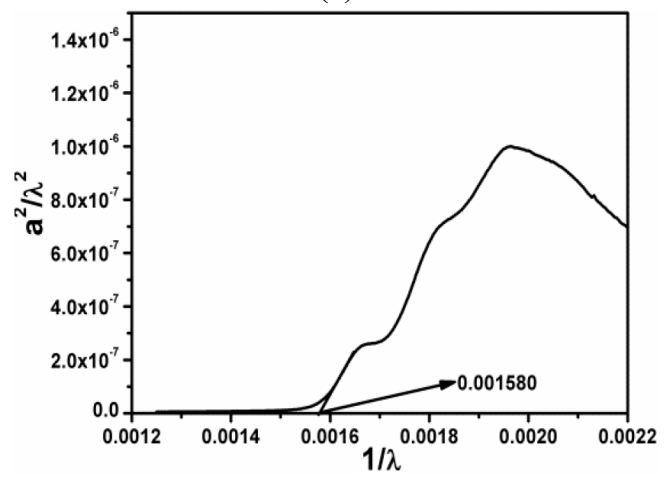

(c)

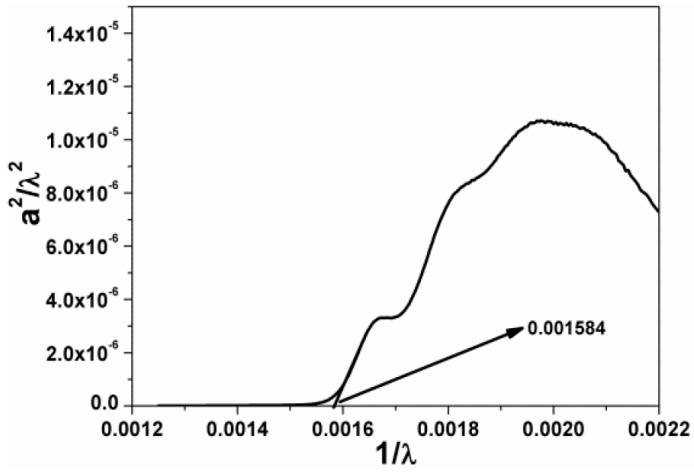

(b)

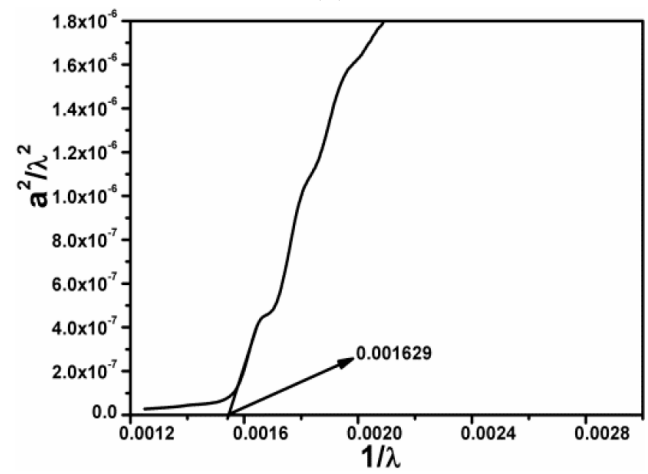

(d)

FIG. 2. Tauc's plot for pure P (a); 3:1 P:N (b); 1:1 P:N (c); 1:3 P:N (d)

\section{References}

[1] Ritesh Tipnis, Darin Laird, Mathew Mathai. Polymer-based Materials for Printed Electronics: Enabling High Efficiency Solar Power and Lighting. Material Matters, 2008, 3 (4), P. 92.

[2] Li G., Vishal Shrotriya, et al. Manipulating regioregular poly (3-hexylthiophene):[6,6]-phenyl-C 61 -butyric acid methyl ester blends?route towards high efficiency polymer solar cells. Journal of Materials Chemistry, 2007, 17, P. 3126-3140.

[3] Mohamad K.A., Afishah Alias, et al. Mixed P3HT/PCBM Organic Thin-Film Transistors: Relation between Morphology and Electrical Characteristics. J. Chem. Chem. Eng., 2014, 8, P. 476-481.

[4] Hummelen(Kees) J.C. Improved fullerene materials for plastic photovoltaics.

http://www.rug.nl/msc/research/groups/molecularMaterialsDevices.

[5] Chirvase D., Parisi J., Hummelen J.C., Dyakonov V. Influence of nanomorphology on the photovoltaic action of polymer-fullerene composites. Nanotechnology, 2004, 15, P. 1317. 